\title{
Long-term phonological knowledge supports serial ordering in working memory.
}

$\operatorname{AUTHOR}(\mathrm{S})$ :

Nakayama, Masataka; Tanida, Yuki; Saito, Satoru

\section{CITATION:}

Nakayama, Masataka ...[et al]. Long-term phonological knowledge supports serial ordering in working memory.. Journal of experimental psychology. Learning, memory, and cognition 2015, 41(5): 1570-1578

\section{ISSUE DATE:}

2015-09

URL:

http://hdl.handle.net/2433/202597

\section{RIGHT:}

This article may not exactly replicate the final version published in the APA journal. It is not the copy of record.; この論文は出版社版でありません。引用の際には出版社版をご 確認ご利用ください。; This is not the published version. Please cite only the published version. 
Long-term phonological knowledge

This is a manuscript for the final submission and may not exactly replicate the final version published in the APA journal (JEP:LMC). It is not the copy of record. The published version is found at http://psycnet.apa.org/psycinfo/2015-08341-001/ 
Running head: Long-term phonological knowledge

Long-term phonological knowledge supports serial ordering in working memory

\author{
Masataka Nakayama ${ }^{1,2}$ \\ Yuki Tanida ${ }^{1,2}$ \\ Satoru Saito ${ }^{1}$
}

1. Graduate School of Education Kyoto University, Yoshida-honmachi, Sakyo-ku, Kyoto 6068501, Japan

2. Japan Society for the Promotion of Sciences, 8 Ichibancho, Chiyoda-ku, Tokyo, 102-8472, Japan

Corresponding Authors:

Masataka Nakayama or Satoru Saito

Graduate School of Education Kyoto University, Yoshida-honmachi, Sakyo-ku, Kyoto 606-8501, Japan.

Telephone: +81757533067

Fax: +81757533066

E-mail: nakayama.masataka.36x@st.kyoto-u.ac.jp or saito.satoru.2z@kyoto-u.ac.jp

Acknowledgements: Part of data was presented at the 10th Annual Conference of the Japan Society for Working Memory, Kyoto, Japan, 18th Conference of the European Society for Cognitive Psychology, and Meeting of the Experimental Psychology Society. This research was supported in part by JSPS KAKENHI Grant Numbers 11J07208 to MN and 25380980 to SS. We 
appreciate Yuya Ishibashi, Megumi Masuda, Yuki Nozaki, and Atsuko Tominaga for data collection. We are also grateful to David Plaut and three anonymous reviewers for helpful comments on the earlier version of the manuscript. 


\begin{abstract}
Serial ordering mechanisms have been investigated extensively in psychology and psycholinguistics. It has also been demonstrated repeatedly that long-term phonological knowledge contributes to serial ordering. However, the mechanisms that contribute to serial ordering have yet to be fully understood. To understand these mechanisms, we demonstrated two effects using triples of Japanese nonwords in immediate serial recall. One, a type of bi-element frequency effect, is a retrograde compatibility effect. Bi-element frequency effects are wellestablished phenomena whereby a two-element sequence (e.g., "ka-re") that frequently appears in a language instantiates better recall of any sequence that includes this element (e.g., "ka-re-sumo"). We demonstrated that bi-element frequency affected both the first (e.g., "ka" for "ka-re"; retrograde compatibility effect) and second part of the sequence, indicating the existence of minicontext representations of two-element sequences. The other effects are the position-element(s) frequency effects, whereby an element (e.g., the mora "ka") that more frequently appears in one position of a sequence (e.g., in the first mora of a word) than in other positions facilitates better recall of that element (i.e., the first mora). The effects demonstrated in this paper indicate the long-term associations of position representations and elements. These effects are discussed in terms of the extensive learning hypothesis, which assumes that phonological structures are learned gradually. Implications for computational models are also discussed.
\end{abstract}

Keywords: serial order control, long-term phonological knowledge, working memory, immediate serial recall, nonword 
Long-term phonological knowledge supports serial ordering in working memory

\section{Introduction}

Serial order control is an important aspect of language processing. Humans learn an enormous number of word forms to refer to any desired concept using a finite repertoire of constituents (e.g., phonemes). This process requires proficiency in dealing with order information. On the one hand, researchers have extensively studied the mechanisms for serial ordering in short-term memory (STM) and speech production, leading to the development of variety of computational models. On the other hand, it has been demonstrated repeatedly that linguistic knowledge contributes to serial ordering in STM and speech production, leading to a deeper understanding of the relationship between STM and long-term memory (LTM) as well as that between language production and acquisition. However, the precise mechanisms of LTM contribution to serial ordering have yet to be fully understood. In the following sections, we review the LTM effects and associated paradigms focusing on three well-studied aspects of phonological structure: bi-element frequency, position-element(s) frequency, and lexical frequency. We then discuss the data still absent from the literature.

The bi-element frequency effect is a phenomenon whereby frequency of a subsequence of two linguistic elements (e.g., letters, phonemes, morae) in a language contributes to recall accuracy of those sequences (particularly in STM tasks) that include the subsequence. For example, bi-mora "ka-re," "re-su," and "su-mo" frequently appear in Japanese and the sequence "ka-re-su-mo" is easily recalled by Japanese speakers. This effect is demonstrated in immediate serial recall (ISR) tasks as an effect of phonotactics in a natural language (e.g., Gathercole, Frankish, Pickering, \& Peaker, 1999; for a review, see Botvinick \& Plaut, 2006). Laboratory 
learning paradigms also demonstrate the effect. In these paradigms, participants learn an artificial language in which a class of bi-elements appeared more frequently than other classes. The acquired knowledge then influences performance in an ISR task (Botvinick, 2005; Majerus, Martinez Perez, \& Oberauer, 2012). Despite the robustness the effects of bi-element frequency, the precise mechanisms of the effects have yet to be investigated.

The position-element(s) frequency effects have typically been investigated in the context of speech production. In English, for example, " $h$ " must occur at the onset of a syllable (Dell, Reed, Adams, \& Meyer, 2000). Even erroneous utterance is constrained by such positional frequency, indicating the presence of implicit knowledge regarding position-element(s) frequency. Dell and colleagues (Dell et al., 2000; Warker \& Dell, 2006) trained participants, having them read aloud nearly 400 lists of four CVC nonwords. Critically, these nonwords were constrained in such a manner that a consonant (e.g., "f") always appeared at a specific position (e.g., coda). Observed speech errors followed the newly learned positional constraints (e.g., "f" is a coda). Such positional constraints in errors have led researchers to implement position representations into models for serial ordering in speech production (Dell, Burger, \& Svec, 1997). Position-element associations are also widely implemented serial ordering mechanisms for STM (for a review, see Hurlstone, Hitch, \& Baddeley, 2014). Thus, it can be inferred that long-term position-element association contributes to STM performance.

However, the broader picture is significantly more complicated. In the Hebb paradigm, a commonly used serial learning paradigm, only limited effects of long-term position-element association is demonstrated. In a typical Hebb paradigm, participants perform a series of ISR tasks, and performance improves when the same list is presented repeatedly (e.g., for every third trial), in contrast to that in non-repeated lists (Hebb, 1961). Using a variant of this paradigm, 
Cumming, Page, and Norris (2003) examined positional transfer effects. Similarly, Hitch, Fastame, and Flude (2005) tested the effects of partial positional repetition (e.g., elements in odd positions are repeated through). These studies demonstrated only limited positional transfer or repetition effects. Instead, repetition of the whole sequence, or a sequence that shares the start of the sequence with the target sequence, has much stronger effects (see also Majerus et al., 2012). Thus, Hebb paradigm results suggest that, in serial learning, "an important role is played by higher-order global information relating to the sequence-as-a-whole" (Hitch et al., 2005, p257). Based on these data, STM models implement long-term knowledge using multiple context sets (Burgess \& Hitch, 2006) or chunk nodes (Page \& Norris, 2009), with each context/chunk representing one whole sequence. In these models, position/order representations are not separated but embedded within each context/chunk for one whole sequence and their association with the corresponding elements. Regarding these as models for word learning, the context/chunk represents the phonological lexicon (Page \& Norris, 2009) and the Hebb effect for sequences of onset-matched repetition can be characterized as a "lexical" or an onset-matched neighborhood frequency effect (Szmalec, Duyck, Vandierendonck, Mata, \& Page, 2009). These effects and the model implementations are distinguished from those of bi-element frequency and position-element(s) frequency.

As we have seen, some crucial aspects have not yet been examined in the literature regarding bi-element frequency and position-element(s) frequency effects. First, mechanisms of the bi-element frequency effect have yet to be investigated. A candidate for a mechanism is a forward chaining in which a preceding element serves as a cue to the next associated element (Lewandowsky \& Murdock, 1989). This direct association from one element to the next could be stored in LTM. Rather than directly associating elements, an additional representation —or 
context representation — can also bind elements together. Elements are associated with a context that supports recall of these elements. Although here we use the term context following Burgess and Hitch (2006), concepts such as chunk (Page \& Norris, 2009), control element (Estes, 1972), and conjunctive representation (Cer \& O’Reilly, 2006) all have a similar function. Extending upon the idea of a whole-list context, if more local mini-contexts for the two adjacent elements also operate at the subsequence level, these contexts would constitute the basis of the bi-element frequency effect. These two mechanisms explain the overall bi-element frequency effect but can be differentiated by a specific prediction on subsequence-level effects. The forward chaining predicts that frequency of a bi-mora (e.g., how often "ka-re" has been previously experienced) only influences the recall of the second part of the bi-mora (e.g., "re"), as the first part is a cue for the second, but the converse is not true. The mini-context account claims that the first (e.g., "ka" for "ka-re") and the second part (e.g., "re" for "ka-re") of a bi-mora are supported by the retained/retrieved mini-context. For example, "ka-re" appears more frequently in Japanese than "ka-ho." If the mini-context for "ka-re" is formed (and associated with "ka" and "re") through long-term learning, then Japanese speakers would recall "ka" in "ka-re" better than "ka" in "kaho." Thus, this retrograde compatibility effect differentiates the forward chaining from the minicontext account. At a whole-sequence level, Botvinick and Plaut (2006; Botvinick, 2005) have reported a retrograde compatibility effect in a behavioral experiment and its simulation using a simple recurrent network. To our knowledge, however, no study has yet examined subsequencelevel retrograde compatibility effects. The first purpose of the current study, therefore, was to test the retrograde compatibility effects to differentiate the forward chaining and mini-contextelement association accounts. Note that both predict bi-element-level rather than whole- 
sequence-level contributions of knowledge. For example, when "ka-re-su-mo" is recalled, frequency of "ka-re" would not influence recall of "su" or "mo."

The second aspect concerns the position-element(s) frequency effect. Position-element(s) frequency effects in speech error indicate long-term position-element associations that are separable from the whole-sequence-level context, whereas the Hebb paradigm only provides limited support for them. One possible explanation for the difference in effects is the difference in measurement (i.e., speech error vs. STM task performance). We nevertheless suspect that measurement is not the main cause of the difference in the effects, as accumulating evidence suggests common serial ordering mechanisms for speech production and serial order memory (e.g., Acheson \& MacDonald, 2009; Nakayama \& Saito, 2014). A plausible explanation may be the extent of training. The speech production paradigm requires participants to read nearly 400 sequences in which the experiment-wide constraints are always satisfied. In contrast, the Hebb paradigm requires participants to recall each (partially) repeated list up to eight times. The number of repetitions is, in fact, sufficient to learn the entire list, but might be insufficient to support the null hypothesis for long-term position-element associations. It is worth noting that limited training may also account for the limited effect of bi-element frequency in the Hebb paradigm (Hitch et al., 2005). In addition, Majerus et al., (2012) demonstrated that the types of to-be-learned material (i.e., digit vs. phoneme) influence laboratory learning, probably reflecting learning history The second purpose of this study was to test the extensive learning hypothesis of position-element associations. If extensive learning could develop position-element associations - in other words, if it could form long-term positional representations — we should detect an effect of position-element associations even in a STM task using natural language materials. Thus, we should find position-specificity in position-element(s) frequency effects. For 
example, when "ka-re-su-mo" is recalled, recall performance of "ka" should be most strongly affected by the frequency of "ka" at this position.

To achieve our two purposes, we conducted two Japanese nonword ISR experiments. Japanese is an ideal language for our purposes because it has many multi-moraic/-syllabic words, which ensure that Japanese speakers have learned associations between within-word position and sublexical element morae ${ }^{1}$. Thus, we used Japanese four-mora nonwords varying four types of phonotactic frequency: mora frequency $(\mathrm{MF})$, bi-mora frequency $(\mathrm{BMF})$, position-mora frequency (PMF), and position-bi-mora frequency (PBMF). BMF is a bi-element frequency and PMF and PBMF are position-element(s) frequencies (see Methods section for details). In these experiments, we examined the subsequence-level effects by conducting position-wise regression analyses of the recall accuracy of each mora rather than the whole-item-level analysis to test the predictions above: retrograde compatibility effects and position-selectivity of positionelement(s) frequency effects. As they share most methods and analyses, we reported these experiments in the same sections.

\section{Experiments $1 \& 2$}

\section{Methods}

Participants. Thirty graduate and undergraduate students from Kyoto University participated in each experiment in exchange for a book coupon worth 1000 yen. Participants in Experiment 1 ranged in age from 19 to 56 year old $(\mathrm{M}=23.7)$, and 17 were female; those in Experiment 2 ranged from 18 to 28 years old $(M=21.0)$, and 21 were female. None participated 
in both experiments. Three additional participants were excluded from the analysis because they failed to complete the experiment. All participants were native Japanese speakers.

Phonotactic frequencies and probabilities. Frequencies were calculated and used for stimuli selection and probabilities were calculated and submitted to the regressions to examine purer effects of the frequencies. At the time of stimuli selection, frequencies were controlled at the item-level (e.g., summed frequency of all morae) rather than at the individual-mora-level because mora-level experimental control might have greatly reduced the number of stimuli. Therefore, we combined post-hoc mora-level statistical control with item-level experimental control as the best method available. MF and BMF were calculated by Tamaoka and Makioka (2004) from a Japanese corpus (Amano \& Kondo, 2000). PMF and PBMF were newly calculated from the same corpus for the current study. MF indicates how often each mora appeared in the corpus (e.g., "ka" appeared 20,530,065 times), irrespective of within-word position of the mora. BMF (i.e., bi-element frequency) indicates how often each bi-mora appeared in the corpus (e.g., "ka-re" appeared 115,601 times). BMF is calculated irrespective of its position in a word and BMF effects reflect mini-context representation that is free from the positional representations in a word. PMF (i.e., position-element frequency) was calculated by counting how often a given mora appeared in a given position in four-mora words ${ }^{2}$. For example, the mora "ka" appeared in the first mora position of four-mora words such as "ka-ra-o-ke," "ka-mi-sa-ma" (god), and so on. The frequency values of these words in the corpus $(2588,1431$, and so forth) are added together to calculate the first-position-mora frequency (PM1F) of "ka." The mora "ka" appeared $2,931,456$ times as the first mora of four-mora words and 433,327 times as the fourth mora of four-mora words (e.g., "a-me-ri-ka”). This imbalance/predictability of PMF in each position of "ka" would influence formation of position-mora association and subsequently recall 
performance of "ka" in an ISR task (e.g., "ka" would be better recalled as a first mora than fourth mora). PBMF is calculated, as is PMF, by counting how often a given bi-mora appeared in a given position in four-mora words. One difference between BMF and PBMF is in the position specificity of the frequency for the latter. For example, the bi-mora "ka-re" appeared 19,998 times as a second-third bi-mora of four-mora words (e.g., "wa-ka-re-ru” [separate]) and 180 times as a third-fourth bi-mora of four-mora words (e.g., "ki-du-ka-re" [mental fatigue]). If the bi-mora is represented in a position-specific manner, recall performance of the bi-mora "ka-re," appearing in the second-third mora position, would be higher than that for the third-fourth position. However, if the bi-mora is (also) represented in a position-free or generalized manner, recall accuracy of the bi-mora "ka-re" would (also) reflect total frequency of the bi-mora, irrespective of their presented positions.

Using these frequencies, we calculated new probability variables in order to capture the relatively pure effects of underlying long-term knowledge. They were bi-mora probability (BMPr), position-mora probability (PMPr), and position-bi-mora probability (PBMPr).

BMPr was calculated by dividing BMF by MFs and PBMF. BMF was affected by the frequency of the two ingredient morae that should be controlled. Dividing BMF (e.g., 115,602 for "ka-re" in a nonword "ka-re-su-mo") by MFs (e.g., 20,530,066 and 5,777,890 for "ka" and "re" respectively) produces probability of co-occurrence in that order (e.g., 0.000000000975). This probability was further divided by PBMF (e.g., 1,819 for "ka-re" as first-second bi-mora) producing BMPr (e.g., 0.000000000000536) to examine the effect of generalized/position-free knowledge. The more frequently the bi-mora appeared in other positions, the larger the BMPr became. If serial ordering mechanisms could not generalize the knowledge of BMF in one position to another, the BMPr would consist only of noise and would not demonstrate any effect. 
PMPr was calculated by dividing PMF (e.g., 2,931,457 for "ka" as first mora) by MF (e.g., 20,530,066 for "ka"). This probability (e.g., 0.143) indicates the position-specificity of the appearance of the mora.

PBMPr was calculated by dividing PBMF (e.g., 1819 for "ka-re" as first-second bi-mora) by PMFs (e.g., 2,931,457 and 327,885 for "ka" as first mora and "re" as second mora respectively). Dividing PBMF by ingredient PMFs produces position-specific probability of cooccurrence in that order (e.g., 0.00000000189). All calculations were based on $n+1$ frequency. Materials. A pool of four-mora nonwords was created with the following constraints. All morae were CV morae. Vowels were selected from the Japanese vowel inventory: $a, i, u, e$, or $o$. Similarly, consonants were selected from a set of Japanese consonants: $k, s, s h, t, t s, c h, n, f, h, m$, or $r$. Any legal combination of these consonants and vowels that is written in one Japanese letter was included. Repetition of vowels or consonants within a nonword was avoided. $\log 10(n+1)$ values of the phonotactic frequencies (i.e., MF, BMF, PMF, and PBMF) were calculated for each nonword to allow stimuli selection. For the nonword "ka-re-su-mo," for example, $\log 10(n+1)$ values of MF for each of four morae (i.e., "ka," "re," "su," and "mo") were added together, and $\log 10(n+1)$ values of BMF for each of the three bi-mora (i.e., "ka-re," "re-su," and "su-mo") were added together, and so on. For each experiment, 216 nonwords were selected from the pool based on the item-level phonotactic frequencies (Table 1). The experiments shared only one nonword. In Experiment 1, they were selected in a $2(\mathrm{BMF} ;$ high/low $) \times 2(\mathrm{PMF} ;$ high/low $)$ group. In Experiment 2, they were selected in a $2(\mathrm{BMF} ;$ high/low $) \times 2(\mathrm{PBMF} ;$ high/low) group. The target frequencies (i.e., BMF and PMF in Experiment 1, and PBMF in Experiment 2) were orthogonalized, and the other two frequencies (i.e., MF and PBMF in Experiment 1, and PMF in Experiment 2) were matched among groups using Match software (van Casteren \& Davis, 2007). 
Nonwords in the same group were randomly combined into a three-item list, avoiding repetition of the same mora in the same intra-item position. The same list combinations were used for every six participants, and intra-list order was counterbalanced.

Procedure. Participants began each trial by pressing a button. A fixation stimulus “****” was presented at the center of a computer display (Diamondcrysta RDTI92WLM) for $1000 \mathrm{ms,}$ followed by the visual presentation of three consecutive nonwords. Each nonword remained on screen for $1500 \mathrm{~ms}$ and was read aloud by the participant. A 500-ms gap followed every nonword. After the last gap, a recall cue "????" was presented. All nonwords were written in hiragana, the most commonly used Japanese syllabary. Each hiragana letter corresponded to one mora and all nonwords were readable, although the experimenter recorded all reading errors. Those trials exhibiting reading errors were excluded from the analysis. All visual stimuli were presented in a white MS Mincho font against a black background.

Participants were asked to recall the nonwords by writing down all morae in the order presented. The answer sheets had three rows of four connected boxes on every page. Each box corresponded to one mora and each row to one nonword. Hot Soup Processer (http://hsp.tv/) controlled the experiments running on a DELL Dimension 9150. For each experiment, all participants experienced all four frequency conditions, each of which consisted of 18 trials. The trial order was pseudo-randomized using Mix (van Casteren \& Davis, 2006). Participants could rest freely after every 18 trials. Prior to the experiment, participants signed a consent form, received instructions, and performed four practice trials. The experimental protocol was approved by the research ethics committee for psychological experiments at Graduate School of Education, Kyoto University. 
Long-term phonological knowledge

\section{Data analysis}

In all, $6.5 \%$ of trials were excluded from the subsequent analysis due to reading errors. Using mixed-effects logistic regressions (Jaeger, 2008) in the statistical software package R (R Core Team, 2013), we conducted position-wise analysis on combined data of the two experiments. Although nonwords were selected for a 2 (BMF; high/low) $\times 2(\mathrm{PMF} ;$ high/low) group in Experiment 1 and for a $2(\mathrm{BMF}$; high/low $) \times 2(\mathrm{PBMF}$; high/low) group in Experiment 2 to increase the variance of these frequencies, "non-manipulated" frequencies (i.e., PBMF in Experiment 1 and PMF in Experiment 2) also have some variance (Table 1). To control and even examine these frequency effects, it is desirable to include all types of frequencies/probabilities in the analysis (i.e., to use the same sets of frequencies/probabilities for each experiment). It is also desirable to analyze combined rather than separate data from the two experiments to reveal general patterns and increase the power. Although different participants experienced different sets of items for two experiments, mixed-effects modeling allows us to statistically control these participant and item effects as random effects. We conducted four independent regressions in which the recall accuracy in each within-item position was the dependent variable. Only the morae recalled at the correct position were regarded as correct. Random effects included intercepts for participants and items (see also note of Table A.1). Fixed effects included control variables and predictor variables.

Control variables included list serial position, accuracy in other positions, and MFs. Two variables coded list serial positions. "Primacy" coded the first item as 1 and the other two as 0. "Recency" coded the third item as 1 and the other two as 0 . Accuracy in other positions may have affected accuracy in one position by preventing transpositions to the position. Such an effect could also reflect the effects of whole-item representation (i.e., whole-item representations 
simultaneously contribute to all morae in the same item, generating correlation among morae in terms of accuracy). We did not specify the exact cause, but the effects should be controlled. MFs should be controlled because they can influence accuracy by contributing to memory for morae but not order/position. Frequencies of each of the four morae were included as control variables (i.e., from M1F to M4F, with the number indicating the position of the mora, e.g., M1F means MF of the first mora). $\log 10(n+1)$ values were submitted to regressions.

Predictor variables included BMPr, PMPr, and PBMPr. Mora- or bi-mora-level base 10 logarithms of probabilities were submitted to regressions. Three BMPrs for the three bi-mora were included (i.e., from BM12Pr to BM34Pr, with the number indicating the position). Four PMPrs for the four morae were included (i.e., from PM1Pr to PM4Pr). Three PBMPrs for the three bi-morae were included (i.e., from PBM12Pr to PBM34Pr).

\section{Results and Discussion}

Regression coefficients (z-values) of predictors are reported in Figs. 1-3. Detailed statistics are reported in the Appendix ${ }^{3}$.

Bi-mora probability. The BMPrs demonstrated relatively position-selective effects of retrograde as well as anterograde compatibility effects (Fig. 1). BM12Pr affected accuracy in the first (M1) and second (M2) mora positions. Similarly, BM23Pr affected M3 and BM34 affected M3. The BM12Pr effect on M1 and the BM34Pr effect on M3 are important, as they indicate retrograde compatibility effects ${ }^{4}$. As BMPr effects are detected after controlling position-bimora frequency effects, the effects are free from within-word position representations. Thus, these results support the existence of subsequence-level (i.e., bi-mora-level) context. M4 was not affected by BM34Pr, which might call into question the reliability of the BMPr effect. However, M4 was affected by almost no predictors. A possible reason is discussed later. 
Position-mora probability. The PMPrs demonstrated relatively position-selective effects

(Fig. 2). PM1Pr, PM2Pr, and PM3Pr most affected the accuracy of corresponding mora. In addition, PM1Pr affected M2 and PM4Pr affected M3.

Position-bi-mora probability. The PBMPrs demonstrated relatively position-selective effects (Fig. 3). PBM12Pr affected M2, PBM23Pr affected M3, and PBM34Pr affected M3. Note that PBMPr is a second-order probability, while PMPr is assumed to be a first-order probability. It takes more time to learn second-order correlations than it does to learn first-order ones (Dell et al., 2000; Warker \& Dell, 2006), possibly leading to the weaker PBMPr effects.

The lack of any effects on M4 might be explained by influences of inflection. Japanese (inflectional) words inflect by changing the final mora. Representation of the fourth mora/position (i.e., the final mora of a four-mora word) might be more complex than the other morae/positions. Because of this, the tested phonotactic frequencies might not be a strong predictor in M4. In addition, we calculated PMFs and PBMFs based on the finite form, ignoring inflection (i.e., frequencies of the other forms are combined into that of the finite form). This calculation method was especially noisy in M4. None of the M4-related probability variables predicted accuracy in M4, probably because they were calculated using noisy PMF and/or PBMF estimation. Similarly, an unexpected effect of PMPr4 on M3 accuracy may be explained as false positive because of noise in PM4F calculation.

General discussion

The present two experiments with natural language materials demonstrated the separable effects of bi-element frequency and position-element(s) frequency. Bi-element frequency effects 
showed retrograde as well as anterograde compatibility effects indicating the presence of underlying mini-context representations. Position-element(s) frequency effects showed position selectivity in that frequency in one position contributed mostly to recall of the element(s). Together with the fact that the experiments used nonwords, most of which had no neighborhood word sharing the first three morae, the position selectivity indicates that these frequency effects reflect long-term position-element associations that are independent from the effects of frequency of sequence-as-a-whole.

These subsequence-level frequency effects in natural language materials support the extensive learning hypothesis of subsequence-level learning, as the effects are assumed to reflect long-term language learning. The hypothesis explains the limited position-element(s) (and bielement) frequency effects in the Hebb paradigm in contrast to speech production paradigms. Bielement frequency and position-element(s) frequency are, in fact, learned, but possibly in a gradual manner. Future research is needed to test whether these frequency effects emerge after extensive learning in the Hebb paradigm to confirm the hypothesis. Notably, the Hebb paradigm might help to overcome potential limitations in the present data. Some aspects of the findings, such as the unexpected PM4Pr effects, may contradict the prediction from the hypotheses. As we used natural language materials, inflection or other unknown higher-order frequency effects, inevitably, might have obscured the target effects. The Hebb paradigm, which allows for complete control of to-be-learned structure(s), would compensate for this methodological limitation.

The present data have implications for computational modeling of long-term knowledge. The simple recurrent network can afford implementations of the bi-element and positionelement(s) frequency effects as well as whole-sequence frequency effects (Botvinick \& Plaut, 
2006; Gupta \& Tisdale, 2009). It does not have built-in knowledge, but rather, it acquires knowledge through learning. Other types of models that explicitly implement knowledge (e.g., Burgess \& Hitch, 2006; Hartley \& Houghton, 1996) could also implement the phonotactic frequency effects with precise extension (e.g., additional bi-element contexts and independent positional context). In any case, the present data constrain the modeling. 


\section{Appendix}

\section{List of the stimuli}

\section{Experiment 1}

High bi-mora frequency High position-mora frequency

ka-mu-shi-re, ka-re-su-mo, ku-sa-te-mi, ku-ri-sa-ne, ke-shi-ro-na, ke-su-mi-na, ke-na-su-mo, keha-tsu-ri, ko-mu-ra-chi, sa-ko-mi-ru, sa-chi-ku-ne, sa-hi-ko-tsu, sa-ho-ku-ri, sa-ho-ri-tsu, shi-hoka-ne, se-ma-tsu-ni, so-chi-ku-ma, ta-mu-ko-re, tsu-shi-ro-na, te-su-ki-ro, te-mu-sa-ki, te-mu-shira, na-shi-ho-ke, na-mi-so-re, na-mu-ke-shi, ne-shi-ka-fu, ne-shi-ra-mu, ne-su-ko-ta, ne-ra-su-chi, ha-ko-mu-chi, hi-ra-mu-to, fu-ki-se-ma, fu-ko-re-ma, fu-re-ki-ma, fu-re-ki-mo, he-ra-ni-to, hoka-su-chi, ho-ka-ne-shi, ho-ta-me-su, ho-te-shi-ra, ma-shi-ro-te, ma-chi-ho-ke, ma-tsu-ki-ne, mare-ki-tsu, mi-tsu-so-re, mu-chi-ka-ro, me-su-ra-ni, mo-sa-ki-tsu, mo-na-ku-te, ra-su-me-ni, ri-tsusa-ne, re-ho-ka-su, ro-sa-ki-tsu, ro-mi-se-ka,

High bi-mora frequency Low position-mora frequency ku-sa-chi-ro, ku-sa-te-ho, ku-shi-me-ho, ku-ta-ni-ho, ko-mu-sa-hi, shi-te-mu-ro, su-hi-to-ke, suma-chi-ro, su-ma-ni-ho, so-ni-tsu-me, ta-mu-shi-ho, chi-so-ku-re, chi-ne-ku-sa, chi-ne-ru-ma, chi-ro-ku-me, te-mu-ri-ko, na-mu-ki-se, ne-tsu-ki-ho, ne-mo-ku-sa, no-hi-ta-ke, no-hi-ra-su, noma-su-hi, no-ma-chi-ke, no-mu-ra-se, fu-ka-ri-so, fu-ki-ra-so, fu-ma-chi-ko, ho-se-ru-mi, ma-teki-so, ma-ni-ho-se, ma-re-ki-ho, mu-chi-ka-so, me-ta-ku-hi, me-tsu-ki-so, me-ra-shi-ho, mo-nasu-hi, mo-ra-ku-he, ra-shi-tsu-ho, ra-so-tsu-me, ra-fu-shi-me, ra-mu-ki-no, ra-mu-shi-ko, ri-satsu-no, ri-na-ku-so, ri-na-tsu-me, ri-fu-ka-me, re-na-ku-hi, re-ma-chi-no, ro-se-tsu-ha, ro-se-tsuhi, ro-na-ku-se, ro-ni-tsu-ke, ro-ni-tsu-me, ro-ha-tsu-ke,

Low bi-mora frequency High position-mora frequency ka-shi-he-tsu, ka-ne-ho-su, ka-ru-he-chi, ku-ta-so-ne, ko-ni-re-tsu, ko-ne-hi-ru, ko-ri-me-ha, sane-ho-tsu, sa-mu-ni-te, sa-mo-hi-ku, shi-to-he-na, su-mo-ne-ri, su-ri-me-ha, se-ni-ha-mu, se-nofu-chi, se-mo-fu-ni, se-ra-hi-mo, se-ra-ho-chi, so-ke-ha-ri, ta-su-ho-mi, ta-so-ki-re, ta-no-he-su, chi-na-se-mu, tsu-ka-ho-mi, tsu-ne-ro-ki, tsu-re-so-mi, te-so-ka-ni, te-ho-mi-su, to-ki-he-ma, nake-ho-su, na-se-mo-ki, na-me-ho-chi, ni-sa-fu-te, ni-ta-so-ke, ni-re-ho-ta, nu-to-ka-shi, ne-ki-sota, no-ki-he-su, no-chi-me-fu, no-re-mi-su, ha-ro-tsu-mi, ha-ro-ne-ku, hi-mo-ke-ta, fu-no-ra-te, ma-ki-he-to, ma-ke-ho-tsu, ma-ne-ho-ru, ma-ro-ni-te, mi-re-sa-ko, me-ka-ho-ni, me-to-hi-na, me-na-chi-ru, mo-fu-te-ri, ri-ta-se-mu,

Low bi-mora frequency Low position-mora frequency

ka-chi-mo-he, ki-se-mu-ta, ki-no-he-sa, ku-no-ra-hi, ko-ne-chi-ha, sa-ne-to-hi, sa-me-to-hi, saru-ko-he, sa-ru-ni-he, shi-na-ko-he, shi-ru-ne-ho, su-ha-ro-ne, se-no-tsu-hi, se-hi-ru-ta, ta-he-shiko, te-ko-ra-hi, te-na-ro-hi, te-ni-mu-sa, te-ni-mu-ro, te-ni-ro-ha, ni-se-mu-ko, nu-ha-to-ki, ha-semu-ko, ha-re-mi-so, he-ni-tsu-ko, ma-se-no-fu, ma-ni-se-ho, ma-fu-no-ke, mi-ke-no-fu, mi-nora-he, mu-se-ni-ko, mu-ta-re-so, mu-te-ra-hi, mu-ni-ro-ka, mu-no-chi-ha, me-hi-sa-to, me-hi-rano, me-fu-no-ri, me-ro-tsu-ha, ra-te-no-fu, ra-he-to-shi, ri-se-ha-mu, ri-te-so-na, ri-mu-no-ta, rime-na-ko*, ri-me-ha-ko, ri-me-fu-to, ru-ka-he-chi, ru-ta-hi-ne, ru-na-hi-mo, ru-mi-so-ta, ru-mihe-ta, ru-me-ho-chi, ro-mu-ni-ha, 


\section{Experiment 2}

High bi-mora frequency High position-bi-mora frequency

ka-te-ro-mu, ki-sa-mu-to, ki-su-ne-ra, ki-te-su-ra, ki-ma-ne-fu, ki-me-ro-sa, ki-mo-sa-ne, ki-rote-ma, ku-na-so-re, ku-na-hi-te, ke-to-ma-fu, ko-na-hi-re, shi-ta-ke-fu, shi-ne-tsu-ma, shi-mu-raho, su-me-ro-ka, tsu-ne-ri-mo, te-ka-ri-nu, te-ro-mi-ha, to-fu-mi-se, na-ke-mu-to, na-su-mi-ke, na-re-shi-ho, ne-ku-ra-ho, ne-tsu-sa-ho, ne-fu-ka-shi, ne-mo-ka-fu, no-shi-ma-fu, no-mi-ra-ke, hi-ke-ru-mo, hi-ne-ru-ma, fu-se-ko-ta, ho-su-chi-ra, ma-fu-ki-te, mu-ko-hi-ra, mu-ne-ta-ko, meta-ri-so, mo-ki-tsu-se, mo-shi-re-nu, ra-ki-so-me, ra-shi-fu-ke, ra-ne-su-mi, ra-ho-ke-shi, ra-miko-se, ri-ko-me-na, ri-fu-sa-me, ri-mu-sa-to, ri-mu-to-ke, re-ka-hi-so, re-to-ma-hi, re-fu-na-shi, re-mi-tsu-ko, ro-ta-mi-se, ro-mu-shi-ke,

High bi-mora frequency Low position-bi-mora frequency ka-hi-so-re, ka-hi-no-mu, ka-fu-chi-re, ka-mu-chi-re, ku-so-te-mi, ku-te-ri-ma, ku-ho-sa-re, kuho-me-ri, ke-su-chi-ro, ke-ni-ho-ma, ke-no-ri-mu, ke-ha-chi-mu, ke-ma-chi-no, ko-fu-sa-re, sahi-ko-ne, sa-hi-no-mu, shi-fu-na-ke, su-hi-ka-te, su-hi-ka-ro, su-hi-ko-me, su-hi-ro-ta, su-me-chiro, se-fu-mi-to, chi-ko-fu-me, chi-ha-so-re, tsu-sa-ke-ni, tsu-sa-ri-mo, tsu-ni-ho-ma, tsu-hi-mo-ra, tsu-ho-ka-me, tsu-ho-ke-ni, tsu-ro-na-ke, to-hi-ka-mu, na-me-su-chi, ni-fu-se-ka, ni-me-su-ro, ne-fu-mi-ka, ne-fu-mi-to, no-hi-ka-mu, no-mu-sa-re, no-mu-se-ri, ma-nu-ki-te, ma-ho-ku-te, muke-ho-shi, mo-sa-chi-ke, ra-ki-fu-se, ra-ki-mu-to, ra-ne-ku-hi, ra-fu-te-mi, ra-mi-ho-ke, ru-maho-ke, re-fu-so-chi, re-ho-ki-tsu, re-mu-ho-shi,

Low bi-mora frequency High position-bi-mora frequency ka-se-mu-ni, ka-mi-he-no, ki-no-he-mu, ki-ho-mu-ta, ki-mu-na-te, ko-ni-mu-te, sa-mi-ke-ho, shika-ho-nu, shi-ne-mu-ha, su-na-ho-re, su-ne-ro-ka, su-no-ra-hi, se-no-ha-mi, se-mu-ta-ni, so-kena-hi, so-na-te-hi, so-ru-ne-chi, so-ru-me-na, ta-he-mi-ku, ta-mi-ke-so, chi-me-na-ro, te-sa-fu-no, te-ri-mu-no, to-ne-mu-ha, to-ra-he-shi, ni-so-me-ha, ni-ta-he-ko, ni-fu-so-ta, nu-to-me-sa, no-rase-hi, no-ra-me-hi, ha-mu-ni-ke, hi-mo-ne-ka, hi-ru-so-ke, fu-mi-te-so, he-chi-ru-mo, he-ni-to-sa, ma-ki-he-no, ma-su-he-ko, ma-su-he-no, ma-ni-so-ke, mu-shi-he-ka, mu-se-ho-chi, mu-te-so-ka, mu-te-hi-no, mu-ni-se-ho, mu-ni-te-ka, me-ro-ha-su, mo-hi-ke-sa, ra-ke-ho-tsu, ra-te-hi-mo, rato-he-shi, ri-mu-ha-te, ri-me-na-ko*,

Low bi-mora frequency Low position-bi-mora frequency ka-mu-he-to, ku-so-he-ma, ku-he-so-ta, ke-fu-ni-ma, ke-ho-sa-ni, ke-ho-chi-su, ko-he-ma-ni, sani-mu-te, sa-he-ni-tsu, shi-fu-na-te, su-hi-ne-to, su-he-ta-ki, se-ha-mi-no, se-hi-na-ro, se-ho-ruchi, ta-mu-he-ko, chi-mo-ke-fu, tsu-sa-ni-re, tsu-sa-he-no, tsu-so-ke-ni, tsu-so-ni-re, tsu-so-hame, tsu-ho-ni-ke, tsu-ho-mi-ka, te-fu-ni-ma, to-nu-re-ka, na-so-he-ru, na-te-mi-fu, na-mo-hi-se, ni-he-ta-ro, ni-ma-te-fu, ni-mu-ho-ra, ni-mu-ro-ha, ne-ho-ta-ki, ne-ro-ki-fu, no-tsu-he-ri, no-resa-fu, no-re-chi-fu, ha-so-ke-ni, he-so-ni-ma, ho-se-ni-ma, ma-so-ru-ne, mu-ha-so-ni, me-ho-nisu, mo-ke-ra-chi, mo-se-hi-ka, ra-ko-hi-su, ra-se-ho-ni, ra-hi-no-tsu, ri-me-ha-so, re-nu-ko-ta, reho-mi-ta, ro-ha-ni-ke, ro-me-ha-ni,

*Used for both experiments 
Long-term phonological knowledge

Table A.1 
Long-term phonological knowledge 
Table A.1: Results of mixed-effects logistic regressions

The results of the likelihood ratio test reported here are based on the comparison of the models with random intercepts for participants and items. We also conducted likelihood ratio tests in which models include the random slope of the tested fixed effects for participants as recommended by Barr, Levy, Scheepers, and Tily (2013). These additional analyses demonstrated an identical pattern of results.

MF: Mora frequency, BMPr: Bi-mora probability, PMPr: Position-mora Probability, PBMPr: Positionbi-mora Probability, numbers indicate the position. 


\section{References}

Acheson, D. J., \& MacDonald, M. C. (2009). Verbal working memory and language production: Common approaches to the serial ordering of verbal information. Psychological Bulletin, 135(1), 50-68. doi:10.1037/a0014411

Amano, S., \& Kondo, T. (2000). Japanese NTT database series: Lexical properties of Japanese, word-frequency (II). Tokyo: Sanseido [in Japanese].

Barr, D. J., Levy, R., Scheepers, C., \& Tily, H. J. (2013). Random effects structure for confirmatory hypothesis testing: Keep it maximal. Journal of Memory and Language, 68(3), 255-278. doi:10.1016/j.jml.2012.11.001

Botvinick, M. M. (2005). Effects of domain-specific knowledge on memory for serial order. Cognition, 97(2), 135-51. doi:10.1016/j.cognition.2004.09.007

Botvinick, M. M., \& Plaut, D. C. (2006). Short-term memory for serial order: a recurrent neural network model. Psychological Review, 113(2), 201-233. doi:10.1037/0033-295X.113.2.201

Burgess, N., \& Hitch, G. J. (2006). A revised model of short-term memory and long-term learning of verbal sequences. Journal of Memory and Language, 55(4), 627-652. doi:10.1016/j.jml.2006.08.005

Cer, D., \& O'Reilly, R. (2006). Neural mechanisms of binding in the hippocampus and neocortex: Insights from computational models. In H. D. Zimmer, A. Mecklingber, \& U. Lindenberger (Eds.), Handbook of binding and memory: Perspectives from cognitive neuroscience (pp. 193-220). Oxford, England: Oxford University Press.

Cumming, N., Page, M., \& Norris, D. (2003). Testing a positional model of the Hebb effect. Memory, 11(1), 37-41. doi:10.1080/09658210143000452

Dell, G. S., Burger, L. K., \& Svec, W. R. (1997). Language production and serial order: a functional analysis and a model. Psychological Review, 104(1), 123-47.

Dell, G. S., Reed, K. D., Adams, D. R., \& Meyer, A. S. (2000). Speech errors, phonotactic constraints, and implicit learning: a study of the role of experience in language production. Journal of Experimental Psychology: Learning, Memory, and Cognition, 26(6), 1355-1367.

Estes, W. K. (1972). An associative basis for coding and organisation in memory. In A. W. Melton \& E. Martin (Eds.), Coding processes in human memory (pp. 161-190). Washington, DC: V.H. Winston \& Sons.

Gathercole, S. E., Frankish, C. R., Pickering, S. J., \& Peaker, S. (1999). Phonotactic Influences on Short-Term Memory. Journal of Experimental Psychology: Learning, Memory, and Cognition, 25(1), 84-95. 
Gupta, P., \& Tisdale, J. (2009). Does phonological short-term memory causally determine vocabulary learning? Toward a computational resolution of the debate. Journal of Memory and Language, 61(4), 481-502. doi:10.1016/j.jml.2009.08.001

Hartley, T., \& Houghton, G. (1996). A linguistically constrained model of short-term memory for nonwords. Journal of Memory and Language, 35, 1-31.

Hebb, D. O. (1961). Distinctive features of learning in the higher animal. In J. F. Delafresnaye (Ed.), Brain mechanisms and learning (pp. 37-46). New York: Oxford University Press.

Henson, R. N. A. (1998). Short-term memory for serial order: the Start-End Model. Cognitive Psychology, 36(2), 73-137. doi:10.1006/cogp.1998.0685

Hitch, G. J., Fastame, M. C., \& Flude, B. (2005). How is the serial order of a verbal sequence coded? Some comparisons between models. Memory, 13(3-4), 247-258. doi:10.1080/09658210344000314

Hurlstone, M., Hitch, G., \& Baddeley, A. (2014). Memory for serial order across domains: An overview of the literature and directions for future research. Psychological Bulletin, 140(2), 339-373. doi:10.1037/a0034221

Jaeger, T. F. (2008). Categorical Data Analysis: Away from ANOVAs (transformation or not) and towards Logit Mixed Models. Journal of Memory and Language, 59(4), 434-446. doi:10.1016/j.jml.2007.11.007

Lewandowsky, S., \& Murdock, B. B. (1989). Memory for serial order. Psychological Review, 96(1), 25-57. doi:10.1037//0033-295X.96.1.25

Majerus, S., Martinez Perez, T., \& Oberauer, K. (2012). Two distinct origins of long-term learning effects in verbal short-term memory. Journal of Memory and Language, 66(1), 3851. doi:10.1016/j.jml.2011.07.006

Nakayama, M., \& Saito, S. (2014). Within-word serial order control: Adjacent mora exchange and serial position effects in repeated single-word production. Cognition, 131(3), 415-430. doi:10.1016/j.cognition.2014.03.002

Otake, T., Hatano, G., Cutler, A., \& Mehler, J. (1993). Mora or Syllable? Speech Segmentation in Japanese. Memory \& Cognition, 32, 358-378.

Page, M. P. A., \& Norris, D. G. (2009). A model linking immediate serial recall, the Hebb repetition effect and the learning of phonological word forms. Philosophical Transactions of the Royal Society of London. Series B, Biological Sciences, 364(1536), 3737-53. doi:10.1098/rstb.2009.0173

R Core Team. (2013). R: A language and environment for statistical computing. Vienna, Austria: R Foundation for Statistical Computing. 
Stemberger, J. P. (1990). Wordshape errors in language production. Cognition, 35, 123-157.

Szmalec, A., Duyck, W., Vandierendonck, A., Mata, A. B., \& Page, M. P. a. (2009). The Hebb repetition effect as a laboratory analogue of novel word learning. Quarterly Journal of Experimental Psychology, 62(3), 435-43. doi:10.1080/17470210802386375

Tamaoka, K., \& Makioka, S. (2004). Frequency of occurrence for units of phonemes, morae, and syllables appearing in a lexical corpus of a Japanese newspaper. Behavior Research Methods, 36(3), 531-547.

Van Casteren, M., \& Davis, M. H. (2006). Mix, a program for pseudorandomization. Behavior Research Methods, 38(4), 584-589.

Van Casteren, M., \& Davis, M. H. (2007). Match : A program to assist in matching the conditions of factorial experiments. Behavior Research Methods, 39(4), 973-978.

Warker, J. A., \& Dell, G. S. (2006). Speech Errors Reflect Newly Learned Phonotactic Constraints. Journal of Experimental Psychology: Learning, Memory, and Cognition, 32(2), 387-398. doi:10.1037/0278-7393.32.2.387 


\section{Footnotes}

1 A mora is a subsyllabic unit including a nucleus onset (CV or CCV), a vocalic nucleus (V), a nasal consonant $(\mathrm{N})$ in the syllabic coda position, and a geminate consonant (Q) (Otake, Hatano, Cutler, \& Mehler, 1993). For example, the two-syllable word bonsai has four morae (CV-N-CV-V: bo-n-sa-i). We tested mora ordering using only CV mora (see Design and Materials sections). This allowed us to minimize the effects of sonority constraints (e.g., Hartley \& Houghton, 1996) and to focus on the phonotactic frequency effects.

2 We confined the calculation of position-mora and bi-mora frequencies to four-mora words because the speech production literature indicates that word shape representations may contain word length information (Stemberger, 1990). In addition, the serial ordering mechanism in STM has been shown to code position information by relative distance not only to the start but also to the end of the sequence (Henson, 1998). Therefore, we assumed that four-mora words have length-unique position representations. It is worth noting that we also conducted regression analyses, as reported in the Results and Discussion section, but using frequencies calculated from all lengths of words. In these analyses, some effects were reduced to non-significant. This may indicate the unreliability of the effects, but is also compatible with the length-unique position representations hypothesis. Frequencies in other lengths might be mere noise for a measure of length-unique position representations.

3 Although we discuss the results of the z-values and their significance, almost identical results were obtained by likelihood ratio tests comparing the full model to a model without the tested variable (c.f., Appendix).

4 The lack of significant effect of BM23Pr on M2 may be explained by a grouping strategy. Participants might have subjectively grouped nonwords into two-by-two groups, which might be 
the natural grouping method for four-mora nonwords. Our unpublished data (Saito, Tanida \& Nakayama, 2014) showed that bi-element frequency effects in an ISR paradigm interacted with temporal grouping. Specifically, there were bi-mora frequency effects within a temporal group, but they were reduced or even eliminated at the group boundary (i.e., when temporal pause separated the bi-mora). In addition, the reduction by pause showed asymmetric effects for the anterograde and retrograde (compatibility) effects, with the latter being affected to a greater extent by the pause. The subjective grouping in the present experiments might have reduced the BM23Pr effect on M2, just as temporal grouping did in Saito et al. (2014). 
Long-term phonological knowledge

Table 1: Psycholinguistic variables of stimuli for Experiments 1 and 2

Added $\log 10(n+1)$ values for each frequency are described. See the main text for detailed

information. MF: Mora frequency, BMF: Bi-mora frequency, PMF: Position-mora frequency,

PBMF: Position-bi-mora frequency

\begin{tabular}{|c|c|c|c|c|c|c|c|c|c|c|c|c|c|}
\hline \multicolumn{7}{|c|}{ Experiment 1} & \multicolumn{7}{|c|}{ Experiment 2} \\
\hline & & & $\mathrm{MF}$ & BMF & PMF & PBMF & & & & $\mathrm{MF}$ & BMF & PMF & PBMF \\
\hline \multirow{8}{*}{$\begin{array}{l}\text { High } \\
\text { BMF }\end{array}$} & & Min & 26.9 & 14.0 & 22.0 & 4.1 & \multirow{7}{*}{$\begin{array}{l}\text { High } \\
\text { BMF }\end{array}$} & \multirow{4}{*}{$\begin{array}{l}\text { High } \\
\text { PBMF }\end{array}$} & Min & 26.4 & 12.5 & 19.3 & 5.1 \\
\hline & High & $\operatorname{Max}$ & 28.1 & 15.3 & 23.8 & 8.1 & & & Max & 27.9 & 13.9 & 22.6 & 7.8 \\
\hline & PMF & Mean & 27.4 & 14.4 & 22.5 & 6.4 & & & Mean & 27.3 & 13.0 & 21.4 & 6.0 \\
\hline & \multirow{5}{*}{$\begin{array}{l}\text { Low } \\
\text { PMF }\end{array}$} & SD & 0.3 & 0.3 & 0.4 & 1.0 & & & SD & 0.3 & 0.4 & 0.7 & 0.7 \\
\hline & & Min & 26.9 & 14.0 & 19.0 & 4.1 & & \multirow{4}{*}{$\begin{array}{l}\text { Low } \\
\text { PBMF }\end{array}$} & Min & 26.5 & 12.5 & 19.2 & 0.3 \\
\hline & & $\operatorname{Max}$ & 28.2 & 15.2 & 21.0 & 8.2 & & & $\operatorname{Max}$ & 27.9 & 13.9 & 22.7 & 3.0 \\
\hline & & Mean & 27.4 & 14.4 & 20.5 & 6.4 & & & Mean & 27.3 & 13.0 & 21.4 & 2.0 \\
\hline & & SD & 0.3 & 0.3 & 0.5 & 1.0 & \multirow{9}{*}{$\begin{array}{l}\text { Low } \\
\text { BMF }\end{array}$} & & SD & 0.3 & 0.4 & 0.7 & 0.9 \\
\hline \multirow{8}{*}{$\begin{array}{l}\text { Low } \\
\text { BMF }\end{array}$} & \multirow{8}{*}{$\begin{array}{l}\text { Low } \\
\text { PMF }\end{array}$} & Min & 26.9 & 9.7 & 22.0 & 4.1 & & \multirow{4}{*}{$\begin{array}{l}\text { High } \\
\text { PBMF }\end{array}$} & Min & 26.4 & 8.5 & 19.2 & 5.0 \\
\hline & & Max & 28.2 & 11.0 & 23.8 & 8.1 & & & Max & 27.9 & 10.5 & 22.7 & 7.8 \\
\hline & & Mean & 27.4 & 10.6 & 22.5 & 6.4 & & & Mean & 27.3 & 10.0 & 21.4 & 6.0 \\
\hline & & SD & 0.3 & 0.3 & 0.4 & 1.0 & & & SD & 0.3 & 0.4 & 0.7 & 0.7 \\
\hline & & Min & 26.8 & 9.7 & 19.0 & 4.1 & & \multirow{4}{*}{$\begin{array}{l}\text { Low } \\
\text { PBMF }\end{array}$} & Min & 26.4 & 8.4 & 19.2 & 0.3 \\
\hline & & $\operatorname{Max}$ & 28.1 & 11.0 & 21.0 & 8.1 & & & Max & 27.9 & 10.5 & 22.7 & 3.0 \\
\hline & & Mean & 27.4 & 10.6 & 20.5 & 6.4 & & & Mean & 27.3 & 10.0 & 21.4 & 2.0 \\
\hline & & SD & 0.3 & 0.3 & 0.4 & 1.0 & & & SD & 0.3 & 0.4 & 0.7 & 0.9 \\
\hline
\end{tabular}




\section{Figure Captions}

Figure 1.

Bi-mora probability effects.

Vertical axis indicates coefficient (z-value) of the probability variables. Horizontal axis indicates intra-item serial position of the morae whose accuracy is predicted. Darker bars indicate effects of bi-mora probabilities of earlier position. Dashed line indicates significance threshold (i.e., 1.96).

Figure 2.

Position-mora probability effects.

Vertical axis indicates coefficient (z-value) of the probability variables. Horizontal axis indicates intra-item serial position of morae whose accuracy is predicted. Darker bars indicate effects of position-mora probabilities of earlier position. Dashed line indicates significance threshold (i.e., 1.96).

Figure 3.

Position-bi-mora probability effects.

Vertical axis indicates coefficient (z-value) of the probability variables. Horizontal axis indicates intra-item serial position of morae whose accuracy is predicted. Darker bars indicate effects of position-bi-mora probabilities of earlier position. Dashed line indicates significance threshold (i.e., 1.96). 
Figure 1.

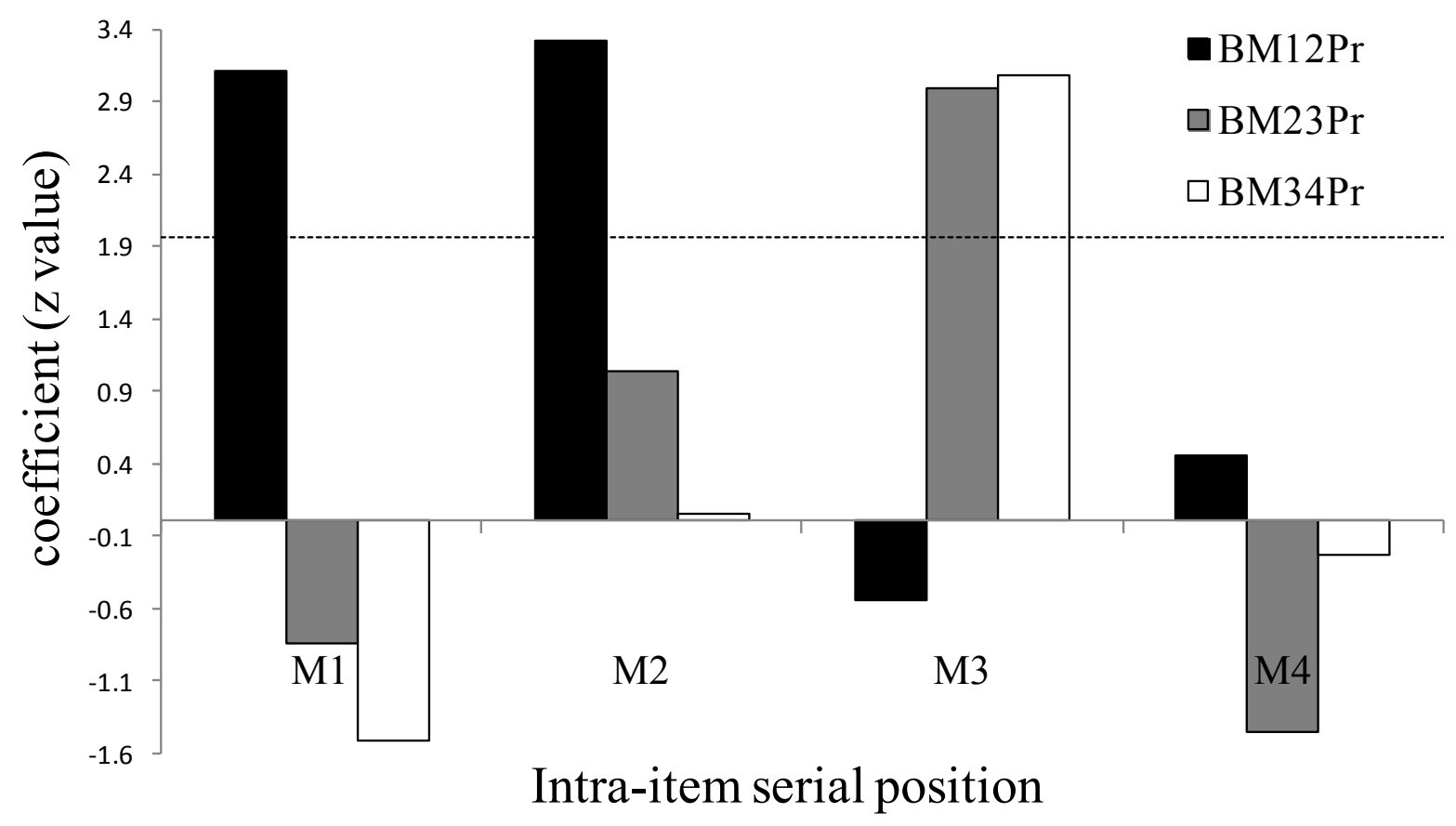


Figure 2.

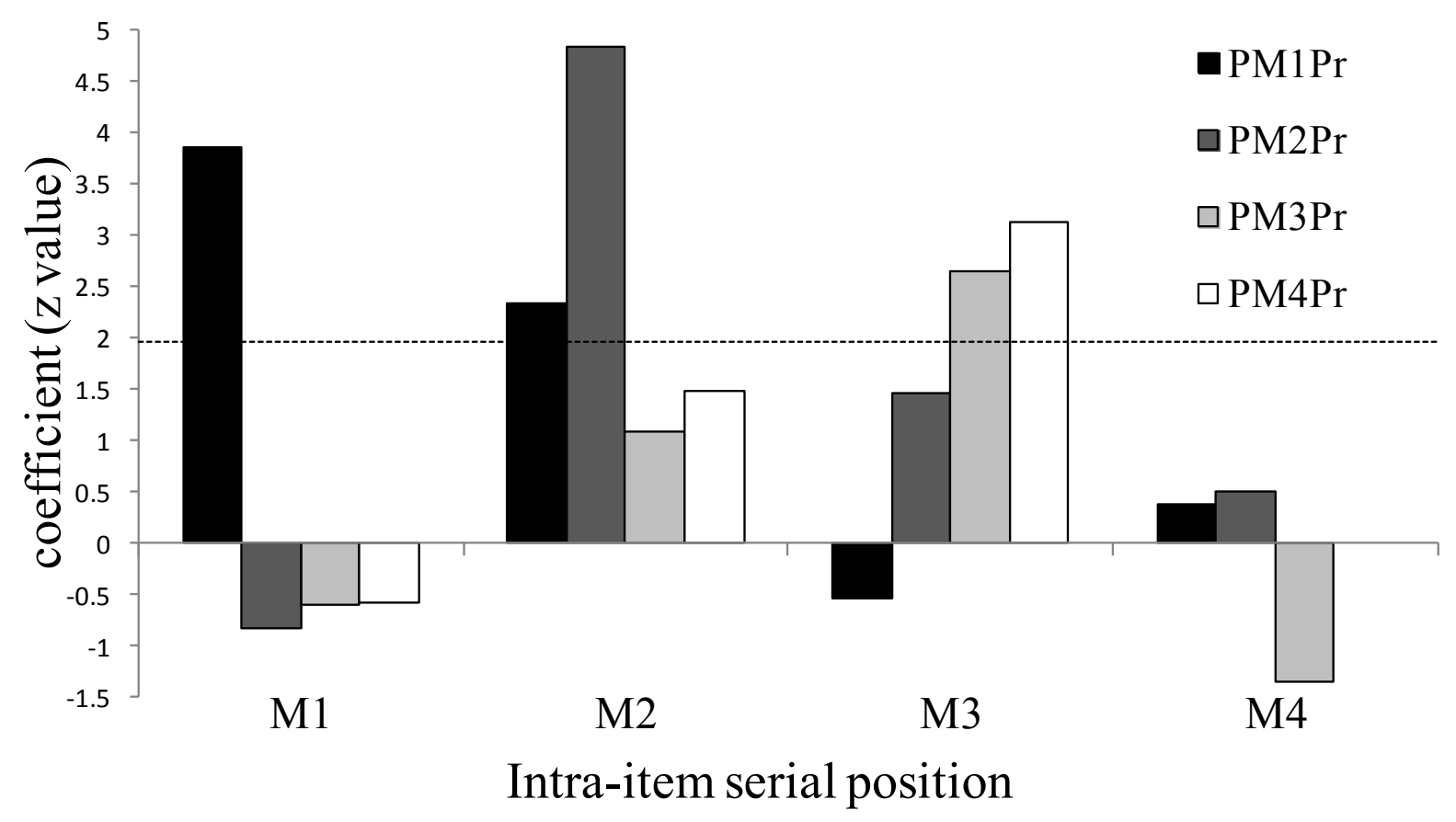


Figure 3.

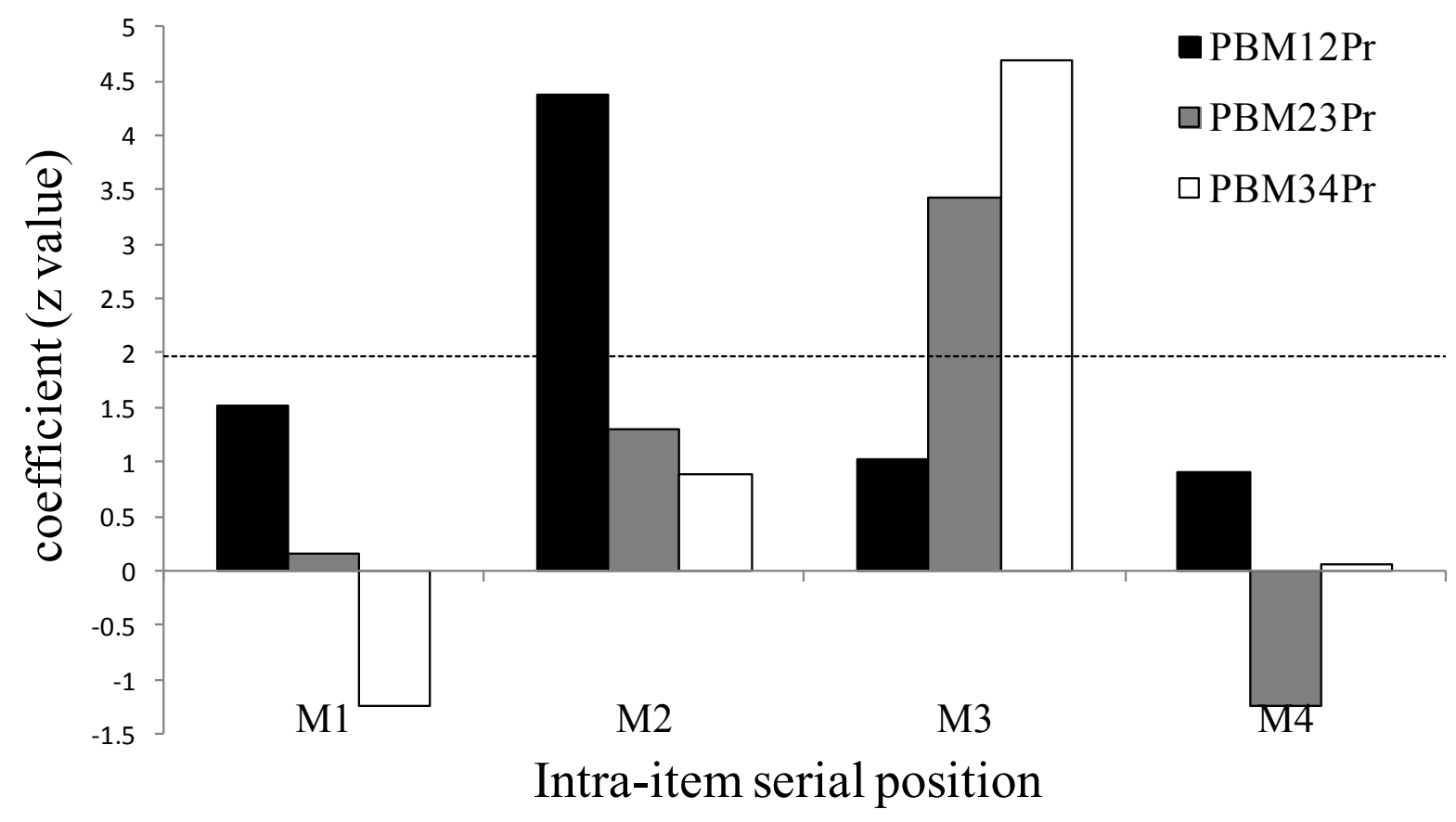

